Voix et Images

volxetimages

\title{
Territoires et lieux du pouvoir poétique
}

\section{Luc Bonenfant}

Volume 32, numéro 2 (95), hiver 2007

Féminin/Masculin. Jeux et transformations

URI : https://id.erudit.org/iderudit/016318ar

DOI : https://doi.org/10.7202/016318ar

Aller au sommaire du numéro

\section{Éditeur(s)}

Université du Québec à Montréal

\section{ISSN}

0318-9201 (imprimé)

1705-933X (numérique)

Découvrir la revue

Citer ce compte rendu

Bonenfant, L. (2007). Compte rendu de [Territoires et lieux du pouvoir poétique]. Voix et Images, 32(2), 139-144. https://doi.org/10.7202/016318ar d'utilisation que vous pouvez consulter en ligne.

https://apropos.erudit.org/fr/usagers/politique-dutilisation/ 


\author{
P O ÉS I E \\ Territoires et lieux du pouvoir poétique \\ $++$
}

LUC BONENFANT

Université du Québec à Montréal

Il y a quelque ironie à signer une première chronique après la parution récente de l'essai de Pierre Bayard, Comment parler des livres que l'on n'a pas lus? Suivant l'argument de Bayard, le chroniqueur parlera forcément mal des livres qu'il a lus puisque «tout écrivain qui a discuté un peu longuement avec un lecteur attentif, ou lu un article assez long à son sujet, connaît cette expérience d'inquiétante étrangeté où il se rend compte de l'absence de correspondance entre ce qu'il a voulu faire et ce qui en a été compris ${ }^{1}$ ». Pire, le chroniqueur parlera même de livres qu'il n'a pas lus, «tout livre dont nous parlons [étant] un livre-écran" fabriqué par "nos fantasmes et nos illusions ${ }^{2}{ }^{»}$. Ceci, bien sûr, ne signifie nullement qu'il faut s'empêcher de parler des livres. De manière provocante, Bayard note d'ailleurs qu'«il est tout à fait possible d'avoir un échange passionnant à propos d'un livre que l'on n'a pas lu, y compris, et peut-être surtout, avec quelqu'un qui ne l'a pas lu non plus ${ }^{3}$ ». Tout dialogue littéraire ne peut donc se réaliser sans afficher en même temps une nécessaire subjectivité.

C'est pourquoi je ne parlerai ici que de recueils que j'ai aimés. Concession rhétorique d'un chroniqueur ne souhaitant pas, en signant sa première chronique, se faire trop rapidement d' "ennemis»? Non. Plutôt: parti pris du lecteur en moi, qui préfère parler des livres qu'il a aimés (même s'il ne les a pas «lus »...). Ma prédilection, cette fois, est allée à des poètes qui font la démonstration de leur foi à l'égard du pouvoir de la parole poétique. Intellectuelle (chez Carole Forget et Yannick Renaud), sociale (chez Tony Tremblay) ou formelle (chez Jean-Éric Riopel), l'écriture occupe chez eux des territoires qui n'ont de cesse d'en exacerber la nécessité.

$$
+
$$$$
+++
$$

1 Pierre Bayard, Comment parler des livres que l'on n'a pas lus?, Paris, Minuit, coll. «Paradoxe», 2007, p. 93.

2 Ibid., p. 53 et 54.3 Ibid., p. 14. 
Comme si le vide avait un lieu ${ }^{4}$, de Carole Forget, frappe d'abord par sa facture soignée. Le carton épais de la couverture, couleur de sable foncé, est orné d'une bande cousue de fil rouge qui donne à lire les informations bibliographiques pertinentes. Ouvrir un si beau livre dispose au plaisir intellectuel de la lecture. Cette sobriété extérieure du livre est aussi celle, typographique, des poèmes qui ne comptent que cinq ou six vers toujours courts et disposés en une ou deux strophes. Frugale, la parole poétique va à l'essentiel en interrogeant le monde à partir de photographies qui inaugurent les sections respectivement intitulées "Abord», «Entre» et «Parages». On aura compris, en lisant ces titres, que le recueil dit constamment les périphéries. D'une manière presque chirurgicale, la voix des poèmes «habite le seuil/avec précision» (51). Les lieux évoqués la mènent à assumer la fragilité du monde et de ses contours puisque éviter les chancellements et les rebords ne permet pas d'assurer la solidité: "s'assure-t-on d'une présence/à la fixer au milieu/comme si/à en faire le tour/on portait/le centre en soi» (19). Notons au passage que les poèmes de Forget ne comportent ni majuscule initiale au vers, ni point terminal. Les marques assurées de la syntaxe régulière ont été effacées au profit d'une écriture qui avère l'esthétique du minuscule en ne coupant pas ses mots des blancs qui les prolongent. Aucune évidence ici, sauf peut-être celle de la constance des déplacements dans un horizon qui ne manque pas de montrer qu'«un centre/en cache toujours un autre» (24). La marche dans le monde - qui est aussi une marche vers le monde - redit le caractère intangible des moments dysphoriques: «fondue à la matière/d'une impression/je tiens debout/l'immensité s'appuie/sur moi/me tourne le dos» (23): le retournement rend impossible un recours concret au monde. Est-ce pour cette raison que le sujet assume la disparition à laquelle il semble voué, alors que son propre souvenir le précède: «souvent/je tourne la tête/surveille/dans mon dos/pour savoir/ce qui disparaît avec moi» (38)? La disparition des balises sûres et résolues traduit la précarité d'une destinée dont l'expédient reste la solitude d'un espace intérieur où «hors de tout écho/j'avance sur les traces/de l'homme»(57). Comme si le vide avait un lieu accepte la passivité inhérente à l'attente, même si son dernier poème engage un lieu plus ou moins actif de continuation: "ultime espace blanc/le dernier pas/inlassablement parcouru/ juste en face/une ouverture/sur l'improbable» (64). Il y a finalement espoir même s'il se trouve repoussé dans les suites d'une interrogation future qui semble être celle, autotélique, de la poésie elle-même.

Méditation sur le deuil, La disparition des idées ${ }^{5}$ de Yannick Renaud est d'une belle et grande placidité. La disparition de l'être aimé s'y vit sur le mode calme et doux d'une sentence qu'il s'agit de comprendre en vue de «ranger les vies comme on archive des souvenirs. Par la fin. Avec méthode» (13). Finement ciselée jusque dans les ellipses qui laissent surgir les pensées les plus fortes, la phrase poétique de Yannick Renaud ne tombe jamais dans le piège de la sentimentalité. La démarche est ici réflexive; le processus de deuil, intellectualisé. Il y a pourtant tout sauf de la

$$
++
$$

4 Carole Forget, Comme si le vide avait un lieu, Outremont, Éditions du passage, 2006, 64 p. Renaud, La disparition des idées, Montréal, Les Herbes rouges, 2006, 56 p.

5 Yannick 
froideur dans le constat. Chaleureuse, la voix poétique s'oppose à la rigidité des monuments pourtant dédiés aux morts, comme ces «cathédrales [qui] ne disent rien de plus que le temps sur leurs pierres» (22), préférant dévoiler le caractère sensible d'une perte dont la conscience attise inlassablement le souvenir, malgré la réalisation que «se souvenir, [est] plus difficile que de ne pas se souvenir» (26). À cette incarnation, qui fonde la force des poèmes, s'ajoute une humilité constante. Le poète sait qu'il ne sait pas quand il écrit que "comparer les guerres c'est prétendre tout connaître» (53). La voix lyrique est aussi mature que modeste, et s'il reste quelque chose de romantique dans le propos, c'en est donc la part la moins hautaine: un Victor Hugo n'aurait jamais pu écrire que «la vue d'un océan confirme la simplicité des peines» (16). On se prend même, en cours de lecture, à penser à l'écriture humble et retirée de Jacques Brault, sans que Renaud y perde nécessairement au change. La syntaxe du recueil pointe inexorablement vers le lyrisme fluide d'une voix qui refuse le panache de la créature solitaire et esseulée pour plutôt se faire "sujet en quête ${ }^{6}$ » qui entend que "le paysage est ce que tu portes derrière les yeux " (30). L'accession à la sphère intime de l'Autre atteste une croyance profonde dans le pouvoir plus vaste des mots. Bien qu'il doive "porter un fardeau de chair, une absence au monde » (21), le sujet poétique endosse sa douleur pour mieux professer sa foi ultime en la vie, "sachant que la mort reste encore le privilège des vivants" (54). Chaque poème semble d'ailleurs répondre à la photographie des étangs d'Auschwitz-Birkenau qui orne la couverture, comme pour nous rappeler que l'éternel retour des désastres n'est pas indiscutable. Oui : «les combats cesseront face à la nostalgie des airs d'enfants » (48). Ce second recueil de Yannick Renaud met en place une éthique de la parole où la singularité de l'être rejoint la sphère universelle sur le mode mineur "du transitoire, de l'attente elle-même» (21). Son lieu n'est pas celui des grands espaces à gagner victorieusement. C'est dans l'intimité du retrait et la simplicité de l'effacement que le poète s'autorise de prendre la parole, dans «l'étendue du territoire: le cœur, cette pierre angulaire. Cœur à occuper» (14).

On conviendra aisément que la circonspection de Carole Forget et de Yannick Renaud n'appartient pas au monde de Tony Tremblay, dont le dernier opus, Rock land $^{7}$, utilise un langage où les mots surgissent à coup de métaphores brutes: «et tout d'un coup notre arbre tousse/ébranle tout le monde» (36). Si les thèmes rappellent pourtant ceux de Renaud (la mort, le souvenir, le crépitement des corps), leur traitement diffère grandement, quoique Tremblay convienne aussi qu'«on ne peut plus prétendre/parler au nom du cosmos» (14). Comme en témoigne le réseau lexical de la dureté qui initie le recueil, Rock land est donc au premier chef, dans sa traduction la plus immédiate, le «monde de la roche». Sorte d'art poétique, le poème

$$
++
$$

6 Dominique Rabaté, "Énonciation poétique, énonciation lyrique», Figures du sujet lyrique, Paris, Presses universitaires de France, 1996, p. 65-79. 7 Tony Tremblay, Rock land, Montréal, l'Hexagone, coll. «L'appel des mots», 2006, 95 p. 
liminaire offre d'emblée les pistes de lecture à privilégier: "que rebelle enfant éponge/c'est en morceaux les mots/le poète l'autre en soi/spectacles d'orages/pour toujours/se fracasser/contre les vagues/de ce qui me fait/métaphore» (11). Le fracas et le tourment (du désastre du monde?), l'enfant rebelle et l'altérité (comment ne pas penser à un certain Arthur Rimbaud?), le morcellement et la métaphoricité (dont l'aliénation serait le symptôme le plus visible?): c'est à partir de ces éléments que le poète construit sa prise de parole. Il sait que la séparation entre dieu et l'humain a été consommée; c'est pourquoi «rock land en souffle pur/point au bout des accords du désastre» (14). Certes, le chemin de la découverte est celui de l'intérieur. Seul dans la violence, le poète déçoit les autres, ses amis, accroché qu'il est « au fil/de toutes les folies» (16). La poésie reste un combat contre l'aliénation de ce «sanatorium de la convergence» (61), si bien nommé. La seconde section du recueil, intitulée "La population des dormeurs», est la plus allégorique. Cette population est prise dans l'ombre dont elle ne semble pas savoir sortir, «comme si chaque instant/m'ayant amené ici/n'avait jamais eu lieu» (46). Mais comme le montre le second vers («m'ayant amené ici»), le rapport au monde en est un de solidarité : le poète ne s'exclut pas de cette population. La conscience obstinée qu'il a de la menace imminente du désastre le somme de «les aimer tous» (43). Il lui revient donc de les délier, à tout le moins de leur montrer l'aliénation qui est la leur pour «que le jour s'éteigne enfin sur la fatigue sur tout ce qu'on peut dire qu'on retient pour ne pas faire s'écrouler les édifices exploser les enthousiasmes terroriser les bien-pensants/ devenir une fin de poème» (71). Posant le problème de la création en termes matériels où les mots sont autant de "pierres/sculptées» (19), la voix poétique insiste sur la nécessité pour le poème de n'épargner personne. Le poème fera mal, littéralement, car il grafigne et déchire. Ceci est d'autant plus vrai que le poète est lui-même un Prométhée qui remet sans cesse à la face de l'Autre son refus «de voir/l'impossibilité du monde» (22). Se surprendra-t-on que la dernière section du recueil est intitulée "Zatoichi de Montréal», quand on sait que le personnage est un célèbre combattant japonais qui protège les innocents? Tremblay réaffirme la puissance d'un verbe poétique qui trouve son sens plein une fois replacé dans l'arène du monde, dans la fraternité humaine, transformant du coup les «morceaux de vie» en «morceaux/de poèmes/sans pervertir le dire» (88). Le recueil se termine donc là où il a débuté: dans l'affirmation du pouvoir communautaire de la poésie, dans le refus du «dérisoire de vivre solitaire» (15). Cet engagement poétique passe bien évidemment aussi par la musique à laquelle le titre fait doublement écho: Rock land est aussi le «monde du rock». Est-il naïf d'entendre Paul Verlaine quand on lit:

rock land

de la musique en tout/pour que ça porte porte des concentrations lumineuses (26)?

Je ne crois pas. Parce que les clichés «fin-de-siècle» qui parcourent les poèmes de Tremblay (la musique, l'ivresse, l'évitement du réel) sont récupérés sur le mode brutal de la contemporanéité la plus immédiate. Pour le dire autrement, le poète 
réactive les vieux sèmes de la poésie décadente pour leur donner une plénitude tout actuelle. Téméraire, il résiste aux assauts du cynisme actuel. Au dix-neuvième siècle, on aurait dit qu'il "s'oppose au bourgeois». On se passera difficilement de cette profession de foi envers l'écriture et la parole poétiques, si ce n'est que pour qu'elle nous redise que le poète est comme nous tous: «écrire/ce n'est pas/être plus seul/que les autres/c'est le savoir/beaucoup plus fort» (77). Le territoire de la poésie est ici celui, extérieur, d'un ancrage social affirmé haut et dur.

Bien que différemment, les trois recueils précédents posent la question de la valeur de la poésie, mais aussi celle de son sens. Car enfin, "que vaut l'éthique qui ne procède pas de cet enracinement dans le sensible, de cette aventure du sensible? Que vaut donc l'éthique qui ne court pas le risque du sensible, c'est-à-dire de la perte de sens (entendre à la fois comme signification et direction) ${ }^{8}$ ? " De ce point de vue, la poésie y apparaît bel et bien comme un espace dynamique de cohérence textuelle où l'acte esthétique converge en définitive vers un acte éthique. Il me semble pouvoir affirmer la même chose du travail plus formel de Jean-Éric Riopel qui ne se réduit pas à la dimension ludique d'un formalisme en mal de pirouettes.

S'il y a quelque chose d'un reste de pensée décadente chez Tremblay, Fermeture des livres de comptes ${ }^{9}$ est explicite quant à sa filiation à Hector de Saint-DenysGarneau. Les poèmes de la seconde section du recueil, «Rue Clark», sans doute les plus difficiles à lire à cause, notamment, de la syntaxe disloquée des vers, constituent un véritable hommage au poète de Regards et jeux dans l'espace. C'est donc dans l'humble repli des espaces intimes que la poésie de Riopel s'énonce: «j'entre dans la chambre blanche/- seul/il n'en sortira pas nécessairement quelque chose/peut-être un tas de feuilles en graffitis de neige/mais il faut parfois/entrer dans la chambre blanche/pour écrire» (23). On comprend tout ce que ce poème oppose à une conception tapageuse de la poésie, lui préférant la nécessité de l'isolement. L'espace du poème sera d'ailleurs essentiellement celui de la lecture, que le poète s'approprie véritablement pour construire son propre monde. Un tel projet n'empêche en rien l'autonomie ludique, qui va jusqu'au montage de vingt-sept vers consécutifs tirés de la poésie garnélienne. Lisant Riopel, nous lisons donc Garneau qui nous renvoie finalement à Riopel. Un saisissant effet d'inquiétante étrangeté s'installe tout en indiquant explicitement que la poésie prend souvent la forme d'un dialogue littéraire avec les prédécesseurs, sorte d'«histoire/pour filtrer le siècle déjà $l^{10}{ }^{10}$. Dans «Marguerite», un bel hommage épique au personnage de Marguerite de Roberval, le poète adopte un ton où Gaston Miron semble un moment rencontrer l'univers d'Anne Hébert (qui avait donné un drame théâtral sur le même personnage). Il y aurait encore beaucoup à dire sur les intertextes contenus dans Fermeture

8 Michel Morin, Le murmure signifiant, Montréal, Les Herbes rouges, 2006, p. 18. Morin souligne. 9 JeanÉric Riopel, Fermeture des livres de comptes, Trois-Rivières, Écrits des Forges, 2006, 112 p. 10 Jean-Simon Desrochers, Parle seul, Montréal, Les Herbes rouges, 2003, p. 9. 
des livres de comptes, d'autant que le recueil puise aussi aux ressources du surréalisme et du formalisme. Mais je veux surtout remarquer que Jean-Éric Riopel est un lecteur redoutable et résolument actif. Son écriture travaille à partir des lectures. Les inscriptions livresques du recueil ne sont pas passives; elles évitent totalement le danger de la débilité nostalgique. L'opération poétique instaure une éthique de la lecture dont on pressent bien qu'elle exige en retour quelque chose de nous. Fermeture des livres de comptes appelle en effet la résolution déterminée de celui qui l'aborde. Comment, par exemple, lire les «Passages d'un amour en amer (Paris)»? Cette section est composée de quatorze poèmes qui sont répartis en deux séries intercalées de sept poèmes au titre identique (à l'exception de leur numérotation): "passage d'un amour/un», «en amer/un», «passage d'un amour/deux», «en amer/ deux ", "passage d'un amour/trois», "en amer/trois», etc. Faut-il lire les sept "passage d'un amour» pour ensuite aborder la suite "en amer»? Ou lire le tout en intégrant l'intercalation qui fait écho au titre de la section? La clé me semble résider dans l'épigraphe: «Nous ne faisons, la plupart du temps, qu'effleurer le monde, comme d'un doigt sur la poussière d'un meuble haut. - Pierre-Albert Jourdan " (62). Car il est impossible de seulement effleurer ces textes, dont l'ordonnancement réglé indique peut-être surtout qu'ils demandent qu'on les lise avec une attention méritée.

Je voudrais terminer cette chronique en mentionnant la parution du plus récent numéro de la Revue Le Quartanier. C'est à la maison d'édition du même nom qu'on doit cette excellente revue sur laquelle j'aurai sans doute l'occasion de revenir. Pour le moment, je vous encourage à vous procurer ledit numéro qui contient notamment des textes de Vincent Sabatier et de Bertrand Laverdure, des traductions de Nathalie Stephens qui permettent de découvrir le travail obsessif - mais si beau - de Sina Queyras, ainsi qu'un entretien stimulant de Guillaume Fayard avec Pierre-Yves Macé et Mathieu Larnaudie. S'il faut encore vous convaincre, achetez ce numéro pour au moins savourer le lumineux «Épisodes des Alains ${ }^{11}$ ", texte d'Ālain Farah où Alain G. mange une lasagne avec Alain R. G., qui lui dédicacera sa copie des Gommes (allez savoir...). Mais enfin, ne vous emballez pas, n'y cherchez pas midi à quatorze heures, ne faites pas comme cette «lectrice en train d'étoffer son hypothèse, trop heureuse de s'engouffrer dans les possibles ouverts par cette triple homonymie» (10). Lisez, simplement, parce que le rire et la bonne humeur ne sont pas synonymes de frivolité.

$$
++
$$

11 Àlain Farah, «Épisodes des Alains», Revue Le Quartanier, nº 7, 2006/2007, p. 5-13. 